\title{
OTIMIZAÇÃO DE ROTAS PARA ENTREGA DOS INGREDIENTES DA MERENDA EM ESCOLAS DA CIDADE DE BARRETOS - SP
}

\section{OPTIMIZATION OF ROUTES FOR DISTRIBUTION OF THE INGREDIENTS OF THE SCHOOL MEALS IN SCHOOLS OF THE CITY OF BARRETOS - SP}

\author{
Augusto César Tová - augustoctdj@gmail.com \\ Faculdade de Tecnologia (Fatec) - Bebedouro - SP - Brasil \\ Oswaldo Lazaro Mendes - oswaldo.lazaro@fatecbb.edu.br \\ Faculdade de Tecnologia (Fatec) - Bebedouro - SP - Brasil
}

DOI: 10.31510/infa.v17i2.1028

Data de publicação: 18/12/2020

\begin{abstract}
RESUMO
O presente artigo tem como objetivo encontrar a rota de menor distância para a entrega dos ingredientes da merenda em 46 escolas da cidade de Barretos - SP, visto que um dos objetivos da logística no que tange transportes, visa buscar a menor rota possível para fazer entregas com agilidade e menor custo. Para a execução desse artigo utilizou-se de pesquisa descritiva, exploratória e quantitativa e para a compreensão do problema proposto utilizou-se o Google Maps, para captação das distâncias, com as escolas e duas entradas da cidade. Este trabalho foi desenvolvido simulando o uso de um caminhão e com dois caminhões devido ao tempo. A fim de encontrar uma rota de menor distância a ser percorrida, o trabalho utilizou-se do Método Evolutionary, do Solver, que é um método capaz de solucionar problemas baseado em algoritmos evolucionários. Como resultado em todos os casos a redução foi expressiva em termos das distâncias percorridas, gerando ganhos em economia de combustível e tempo. Com esse estudo conclui-se que é de suma importância a utilização de tecnologias para que se possa diminuir e otimizar as rotas de entrega, e a ferramenta Solver do Excel se mostra muito eficaz neste quesito.
\end{abstract}

Palavras-chave: Otimizar. Solver. Rotas. Método Evolutionary.

\begin{abstract}
This article aims to find the shortest route for the delivery of lunch ingredients to 46 schools in the city of Barretos - SP, since one of the objectives of logistics in terms of transport, aims to seek the shortest possible route to deliver with agility and lower cost. For the execution of this article, descriptive, exploratory and quantitative research was used and to understand the proposed problem, Google Maps was used to capture the distances, with schools and two entrances to the city. This work was developed simulating the use of a truck and with two trucks due to the weather. In order to find a shorter route to be covered, the work used the Evolutionary Method, from Solver, which is a method capable of solving problems based on evolutionary algorithms. As a result, in all cases, the reduction was significant in terms of the distances
\end{abstract}


covered, generating gains in fuel economy and time. With this study it is concluded that the use of technologies is of utmost importance in order to reduce and optimize delivery routes, and the Excel Solver tool is very effective in this regard.

Keywords: Optimize. Solver. Routes. Evolutionary Method.

\section{INTRODUÇÃO}

A otimização é de natureza humana. Desde os primórdios da raça humana, busca-se diminuir nosso empenho e elevar o retorno sobre qualquer trabalho que será feito, sendo que no início de nossas vidas, se restringia apenas em se manter vivo (LOESCH; HEIN, 2017).

Elaborar a roteirização das entregas é de suma importância na logística de transportes. Encontrar a menor rota é essencial para diminuir o tempo e o custo das entregas. E nesse quesito, alguns softwares e suas ferramentas podem auxiliar na busca pela melhor rota.

Segundo Bertaglia (2020), existem muitos elementos no trabalho de coletar e entregar produtos e, através do uso do computador, pode-se diminuir o tempo por meio de simulações, permitindo chegar a soluções de acordo com restrições pré-determinadas.

Para Dornier et al. (2007), a logística tem como base a otimização, em busca de minimizar o custo de seus serviços ou maximizar o serviço frente a uma limitação de orçamento.

Conforme Ballou (2009) antes de tudo, a relevância logística é demonstrada pelo tempo e lugar. Mercadorias e atividades não têm valia se não estiverem em domínio dos consumidores na data (tempo) e no local (lugar) em que eles desejam.

Com o intuito de apresentar o melhor percurso para a distribuição dos itens para fazer a merenda nas escolas de Barretos, buscou-se com o auxílio de uma ferramenta, Solver, apresentar a melhor rota para otimizar a distribuição.

O objetivo geral deste artigo é encontrar a menor rota para reduzir a quilometragem percorrida na entrega de ingredientes para merenda em 46 escolas da cidade de Barretos.

Como objetivo específico buscou-se avaliar o percurso do veículo durante a distribuição dos itens para fazer a merenda escolar e verificar a necessidade de utilização de dois caminhões ao invés de um caminhão, para que as entregas sejam feitas no mesmo dia e também apresentar como o uso da ferramenta Solver pode auxiliar na roteirização. 


\section{FUNDAMENTAÇÃO TEÓRICA}

\subsection{Logística}

A logística abrange a ligação de informações referentes ao recebimento, armazenagem, estoque, embalagem e transporte de materiais e produtos. Assim, é essencial que a sincronia destes não seja afetada com uma logística falha, sendo o transporte, nos procedimentos logísticos, a área de maior relevância (GOULART; CAMPOS, 2018).

De acordo com Novaes (2007), a logística tem como foco satisfazer os desejos dos clientes, através do procedimento de elaborar, planejar e monitorar de forma competente o curso e a armazenagem de mercadorias, assim como as informações e atividades relacionadas, contemplando toda sua cadeia, do início ao fim.

Brandalise (2017), reforça que a logística implica movimentação de atividades e recursos desde o início até o final da cadeia logística, e o transporte é responsável pela transferência, obtendo os fluxos destes por toda a cadeia.

\subsection{Pesquisa Operacional}

Longaray (2017), define a pesquisa operacional como a união de procedimentos que utiliza o método científico para ajudar as pessoas em suas decisões

Segundo Hillier e Lieberman (2013), a pesquisa operacional é utilizada em uma instituição para que ela possa entender a direção e a administração de suas operações.

Hillier e Lieberman (2013), afirmam que a Pesquisa Operacional procura encontrar a "solução ótima" para a amostra que reproduz a adversidade proposta. Em vez de refinar a condição, o propósito é de detectar o melhor rumo a se seguir.

\subsection{Método Evolutionary}

Apresentado por Xie e Steven (1993, apud Lanes;Greco, 2013), o método Evolutionary (Evolutionary Structural Optimization - ESO), fundamentado no Método dos Elementos Finitos (MEF), foi inicialmente projetado por um algoritmo evolucionário, embasado na ideia da adição 
de vazios, ou seja, demonstra noções na exclusão gradual de componentes pouco buscados do domínio no decorrer do procedimento. Esse processo é denominado de hard-kill.

Segundo Foreman (2018), o algoritmo evolucionário é modelado como o desenvolvimento na biologia:

- Desenvolve um grupo de soluções inaugurais ("patrimônio genético"), praticáveis ou impraticáveis;

- Toda solução tem um grau de duração;

- Soluções desenvolvidas por junções são partes escolhidas e a planejadas por duas ou mais soluções;

- As soluções se transformam em novas;

- Produz-se novas soluções com o intuito de ter uma superior;

- Soluções de menor funcionamento são removidas do patrimônio genético ("seleção").

\title{
2.4 Problema do Caixeiro Viajante (PCV)
}

Rebouças (2016, p. 14) conta que:

\begin{abstract}
No início do século XIX, vários problemas envolvendo grafos, intimamente relacionados ao Problema do Caixeiro Viajante (PCV), foram propostos pelo Matemático Irlandês William Hamilton. Um deles, nomeado por Ball como o "Jogo de Hamilton", consistia em determinar uma rota ao longo das arestas de um dodecaedro regular, passando por todos os vértices, sendo cada um visitado uma única vez. Apesar dos problemas propostos, a primeira abordagem formal do PCV foi apresentada por Hassler Witney, em um seminário na Universidade de Princeton, em 1934.
\end{abstract}

Segundo Gaspar-Cunha, Takahashi e Antunes (2012), o Problema do Caixeiro Viajante se define como a vontade de mover-se pela rota mais curta, o vendedor passa apenas uma vez por cada cidade, iniciando de uma cidade e retornando a ela no final do percurso.

Silva e Oliveira (2006), complementam que o PCV é uma amostra comum da questão de otimização. A partir do momento em que se tem um grupo de $\mathrm{n}$ cidades e a distância entre elas, se tem que estabelecer qual rota irá ser percorrida passando por todas as n cidades apenas uma vez voltando ao ponto de origem, em que a soma dessa distância seja a menor possível. 


\title{
3 PROCEDIMENTOS METODOLÓGICOS
}

Gil (2002) explica a pesquisa como "o procedimento racional e sistemático que tem como objetivo proporcionar respostas aos problemas que são propostos". Neste artigo foram feitas pesquisas bibliográficas através de livros, artigos e internet.

A pesquisa deste é artigo é descritiva, e Gil (2002, p. 42), a elucida como:

\begin{abstract}
As pesquisas descritivas têm como objetivo primordial a descrição das características de determinada população ou fenômeno ou, então, o estabelecimento de relações entre variáveis. São inúmeros os estudos que podem ser classificados sob este título e uma de suas características mais significativas está na utilização de técnicas padronizadas de coleta de dados, tais como o questionário e a observação sistemática.
\end{abstract}

O trabalho parte de uma abordagem quantitativa que, conforme Fonseca (2002), voltase ao estilo matemático para retratas os motivos de um acontecimento, as suas ligações entre variáveis etc. Da Fonseca (2016) complementa "quando uma pesquisa se vale desse tipo de método, ela busca analisar a frequência de ocorrência pra medir a veracidade ou não daquilo que está sendo investigado".

O artigo apresenta finalidade de pesquisa aplicada. Cooper e Schindler (2016) estabelecem como uma pesquisa com foco prático em resolver alguma adversidade, isso quer dizer que ela é direcionada para buscar soluções a problemas específicos referente a atividade, performance ou vontade.

Para o desenvolvimento deste artigo foi utilizada a planilha eletrônica Excel, onde foram feitas várias planilhas com a base de endereços de 46 escolas e 2 entradas da cidade de Barretos - SP, além de outras 4 planilhas contendo as 46 escolas em todas elas, e dividindo elas com: entrada 1; entrada 2; entrada 1 e saída(entrada) 2; entrada 2 e saída(entrada) 1.

Após o desenvolvimento das planilhas, usou-se a "função índice" do Excel em cada uma das planilhas para buscar os valores das distâncias (neste trabalho em metros) nas tabelas, para assim usar a "função soma" e detectar qual a distância percorrida. Assim, então, partiu-se para o uso da ferramenta Solver do Excel, para inserir as restrições e determinar que a ferramenta encontrasse o valor mínimo a ser percorrido. E para isso, aplicou-se o Método Evolutionary, que é uma função da ferramenta Solver, com a finalidade de chegar a menor distância a ser percorrida e qual rota deveria ser feita.

Ao obter os resultados, foi feita uma divisão das escolas para que dois caminhões fizessem as entregas cada um partindo de uma das entradas, a fim de diminuir o tempo das 
entregas, visto que, além do tempo para percorrer a rota, soma-se também o tempo para descarregamento.

$\mathrm{Na}$ fase de coleta de dados foi usado o Google Maps, para encontrar e determinar as distâncias, em metros, entre as 46 escolas e as duas entradas para a entrega de merendas da cidade de Barretos.

\section{RESULTADOS E DISCUSSÃO}

Para dar início aos resultados, foram coletados os nomes e endereços das 46 escolas e das duas entradas para poder determinar as distâncias entre elas

O Quadro 1 conta com os nomes das 46 escolas e as 2 entradas da cidade de Barretos SP, usadas para este estudo.

Quadro 1 - 46 escolas e duas entradas da cidade de Barretos - SP

\begin{tabular}{|c|c|c|c|}
\hline & \multicolumn{3}{|c|}{ ESCOLAS } \\
\hline 1 & $\begin{array}{l}\text { E.M. ANA CARVALHO } \\
\text { CASTANHO (EJA) }\end{array}$ & 25 & CEMEI DR. LUIS SPINA \\
\hline 2 & E.M. ANÁLIA FRANCO & 26 & CEMEI HUMBERTO MINARÉ \\
\hline 3 & $\begin{array}{l}\text { E.M. CHRISTIANO CARVALHO } \\
\text { (EJA) }\end{array}$ & 27 & CEMEI OLÁVIO LOPES \\
\hline 4 & $\begin{array}{l}\text { E.M. SÃO FRANCISCO } 5^{\mathrm{a}} \text { A } 8^{\mathrm{a}} \\
\text { SÉRIE (EJA) }\end{array}$ & 28 & CEMEI ROBSON ROSALINO DA SILVEIRA \\
\hline 5 & E.M. PROF. DORIVAL TEIXEIRA & 29 & CEMEI ANNA KERULIS DA SILVEIRA \\
\hline 6 & $\begin{array}{l}\text { E.M. PROF. JOÃO FERREIRA } \\
\text { LOPES } \\
\end{array}$ & 30 & CEMEI ANTONIO DALLA COSTA \\
\hline 7 & E.M. FAUSTO LEX & 31 & CEMEI CLEUZA PEREIRA BARRETO \\
\hline 8 & E.M. JOÃO BARONI & 32 & CEMEI DO BAIRRO RIOS \\
\hline 9 & E.M. LEODETE SILVEIRO JOI & 33 & CEMEI FERNANDA TEIXEIRA DE ALMEIDA \\
\hline 10 & E.M. LUIZA PARASSU BORGES & 34 & CEMEI IRMÃ ELZA MARTHA MARTHÉIA \\
\hline 11 & $\begin{array}{l}\text { E.M. MARLENE CARBONI } \\
\text { PEREIRA (EJA) }\end{array}$ & 35 & $\begin{array}{c}\text { CEMEI IZADORA BEVILÁCQUA DE SOUZA } \\
\text { MERENDA }\end{array}$ \\
\hline 12 & $\begin{array}{l}\text { E.M. MATILDE GITAY DE MELO } \\
\text { (EJA) }\end{array}$ & 36 & CEMEI JOSÉ PEREIRA NEVES \\
\hline 13 & $\begin{array}{l}\text { E.M. PROF. DOROTHÓVIO DO } \\
\text { NASCIMENTO }\end{array}$ & 37 & CEMEI PROF. LUIZ PARO NETO \\
\hline 14 & $\begin{array}{l}\text { E.M. PROF. GIUSEPPE } \\
\text { CARNIMEO (EJA) }\end{array}$ & 38 & CEMEI LUZIA COSTA FERNANDES \\
\hline 15 & $\begin{array}{l}\text { E.M. PROF. LUIZ CASTANHO } \\
\text { FILHO } \\
\end{array}$ & 39 & $\begin{array}{c}\text { CEMEI MÃE COMERCIÁRIA PROFESSORA CÉLIA } \\
\text { REGINA AIELO }\end{array}$ \\
\hline 16 & $\begin{array}{l}\text { E.M. PROFa MARIA ALVES } \\
\text { BARCELOS DE OLIVEIRA }\end{array}$ & 40 & CEMEI D. MARIA FERNANDES RODRIGUES \\
\hline 17 & $\begin{array}{l}\text { E.M. PROF }{ }^{a} \text { OLGA ABI RACHID } \\
\text { MORAES (EJA) }\end{array}$ & 41 & CEMEI ORIVAL LEITE DE MATOS \\
\hline 18 & $\begin{array}{l}\text { E.M. ROTARY CLUB - VILA } \\
\text { MARILIA (EJA) }\end{array}$ & 42 & CEMEI PAULO BELMIRO FERREIRA \\
\hline 19 & E.M. SAGRADOS CORAÇÕES & 43 & CEMEI ROSA DE SANTIS RIBEIRO \\
\hline 20 & $\begin{array}{l}\text { E.M. ZULEICA INÁCIO LOPES } \\
\text { FERRAZ }\end{array}$ & 44 & CEMEI TENENTE AFONSO CAMARA FILHO \\
\hline 21 & $\begin{array}{l}\text { CEMEI ABDALA MEHDE } \\
\text { REZECK }\end{array}$ & 45 & CEMEI VERIDIANA DA SILVA \\
\hline 22 & $\begin{array}{l}\text { CEMEI AMADOR ALVES DE } \\
\text { QUEIROZ }\end{array}$ & 46 & CEMEI ELZA MARIA PITTA BEZERRA \\
\hline
\end{tabular}


|WTEREFGEETECNOLOGGCA

\begin{tabular}{|c|c|c|c|c|}
\hline 23 & CEMEI DO BAIRRO BARONI & $\mathbf{4 7}$ & $\begin{array}{c}\text { Entrada 1 - Av. João Batista da Rocha, 900 - Nova } \\
\text { América }\end{array}$ \\
\hline 24 & CEMEI DO BAIRRO BARONI II & $\mathbf{4 8}$ & $\begin{array}{c}\text { Entrada 2 - Av. Eng. Necker Carvalho de Camargo, } \\
2711 \text { - América }\end{array}$ \\
\hline
\end{tabular}

Fonte: Prefeitura Municipal de Barretos (2020)

\subsection{Utilizando 1 caminhão para as entregas}

$\mathrm{Na}$ Tabela 1 tem-se os resultados das distâncias percorridas entre a entrada 1 com as 46 escolas em sequência, e a rota otimizada.

\begin{tabular}{|c|c|c|c|c|c|c|c|c|c|c|c|c|c|c|}
\hline \multirow{2}{*}{$\begin{array}{l}\text { Início } \\
\text { Escolas }\end{array}$} & \multirow[b]{2}{*}{47} & \multicolumn{12}{|c|}{ Google Maps } & \\
\hline & & 1 & 2 & 3 & 4 & 5 & 6 & 7 & 8 & 9 & 10 & 11 & 12 & \\
\hline Distâncias & & 8500 & 4200 & 1900 & 6800 & 4000 & 4100 & 2300 & 2700 & 5700 & 2400 & 5800 & 1500 & \\
\hline Escolas & 12 & 13 & 14 & 15 & 16 & 17 & 18 & 19 & 20 & 21 & 22 & 23 & 24 & \\
\hline Distâncias & & 7600 & 750 & 5400 & 3300 & 7200 & 7300 & 3000 & 5300 & 2500 & 7200 & 3600 & 190 & \\
\hline Escolas & 24 & 25 & 26 & 27 & 28 & 29 & 30 & 31 & 32 & 33 & 34 & 35 & 36 & \\
\hline Distâncias & & 4600 & 4100 & 5800 & 6000 & 7800 & 6400 & 3200 & 3600 & 6400 & 2800 & 7400 & 9600 & \\
\hline Escolas & 36 & 37 & 38 & 39 & 40 & 41 & 42 & 43 & 44 & 45 & 46 & & & Total \\
\hline Distâncias & & 3000 & 5200 & 4200 & 1700 & 5800 & 1900 & 650 & 4400 & 4400 & 5000 & 6000 & & 213190 \\
\hline
\end{tabular}

\begin{tabular}{|c|c|c|c|c|c|c|c|c|c|c|c|c|c|c|}
\hline Final & & \multicolumn{12}{|c|}{ Método Evolutionary } & \\
\hline Escolas & 47 & 35 & 17 & 7 & 21 & 11 & 31 & 12 & 27 & 40 & 39 & 10 & 23 & \\
\hline Distâncias & & 3000 & 1500 & 1100 & 200 & 2200 & 52 & 1600 & 350 & 3400 & 2000 & 1200 & 700 & \\
\hline Escolas & 23 & 24 & 28 & 16 & 46 & 4 & 37 & 15 & 18 & 5 & 45 & 36 & 9 & \\
\hline Distâncias & & 190 & 2200 & 550 & 700 & 500 & 2900 & 160 & 1100 & 1500 & 550 & 650 & 1300 & \\
\hline Escolas & 9 & 34 & 43 & 41 & 25 & 29 & 14 & 1 & 13 & 33 & 38 & 22 & 19 & \\
\hline Distâncias & & 850 & 1000 & 1100 & 500 & 2800 & 350 & 95 & 350 & 1000 & 550 & 1500 & 130 & \\
\hline Escolas & 19 & 26 & 42 & 2 & 30 & 3 & 32 & 20 & 6 & 44 & 8 & & & Total \\
\hline Distâncias & & 1000 & 2900 & 1000 & 1900 & 750 & 2100 & 550 & 650 & 550 & 700 & 1000 & & 52927 \\
\hline
\end{tabular}

Fonte: elaborada pelos autores (2020)

De acordo com a Tabela 1, a distância percorrida após ser otimizada pelo Método Evolutionary foi de 52927 metros, partindo de uma distância de 213190 metros, gerando uma redução de 75,13\% em relação a rota inicial. Após ligar essa rota no Google Maps, o tempo para percorrer a rota é de 177 minutos.

Fazendo o mesmo procedimento, substituindo a entrada 1 pela entrada 2, nota-se na Tabela 2 que a distância em relação a entrada 1 é maior.

Tabela 2 - Distância, em metros, entre a entrada 2 e as 46 escolas

\begin{tabular}{|l|c|c|c|c|c|c|c|c|c|c|c|c|c|}
\cline { 2 - 11 } & \multicolumn{10}{|c|}{ Início } & \multicolumn{10}{|c|}{ Google Maps } \\
\hline Escolas & 47 & 1 & 2 & 3 & 4 & 5 & 6 & 7 & 8 & 9 & 10 & 11 & 12 \\
\hline Distâncias & 7300 & 4200 & 1900 & 6800 & 4000 & 4100 & 2300 & 2700 & 5700 & 2400 & 5800 & 1500 \\
\hline
\end{tabular}


WTIERFEGEETECNOLOGGCA

\begin{tabular}{|l|c|c|c|c|c|c|c|c|c|c|c|c|c|c|} 
Escolas & 12 & 13 & 14 & 15 & 16 & 17 & 18 & 19 & 20 & 21 & 22 & 23 & 24 & \\
\hline Distâncias & & 7600 & 750 & 5400 & 3300 & 7200 & 7300 & 3000 & 5300 & 2500 & 7200 & 3600 & 190 \\
\hline Escolas & 24 & 25 & 26 & 27 & 28 & 29 & 30 & 31 & 32 & 33 & 34 & 35 & 36 \\
\hline Distâncias & 4600 & 4100 & 5800 & 6000 & 7800 & 6400 & 3200 & 3600 & 6400 & 2800 & 7400 & 9600 & \\
\hline Escolas & 36 & 37 & 38 & 39 & 40 & 41 & 42 & 43 & 44 & 45 & 46 & & & Total \\
\hline Distâncias & & 3000 & 5200 & 4200 & 1700 & 5800 & 1900 & 650 & 4400 & 4400 & 5000 & 4600 & & $\mathbf{2 1 0 5 9 0}$ \\
\hline
\end{tabular}

\begin{tabular}{|c|c|c|c|c|c|c|c|c|c|c|c|c|c|c|}
\hline Final & & \multicolumn{12}{|c|}{ Método Evolutionary } & \\
\hline Escolas & 47 & 8 & 44 & 6 & 32 & 3 & 30 & 20 & 28 & 16 & 46 & 4 & 18 & \\
\hline Distâncias & & 1000 & 850 & 750 & 600 & 1600 & 25 & 2600 & 3900 & 550 & 700 & 500 & 2900 & \\
\hline Escolas & 18 & 24 & 23 & 10 & 45 & 9 & 43 & 41 & 25 & 1 & 13 & 29 & 14 & \\
\hline Distâncias & & 800 & 190 & 950 & 1700 & 1700 & 1000 & 1100 & 500 & 2500 & 350 & 950 & 350 & \\
\hline Escolas & 14 & 33 & 38 & 26 & 19 & 22 & 5 & 37 & 15 & 36 & 34 & 27 & 21 & \\
\hline Distâncias & & 650 & 550 & 900 & 1000 & 130 & 1900 & 2300 & 160 & 2600 & 1600 & 1700 & 850 & \\
\hline Escolas & 21 & 7 & 35 & 17 & 12 & 11 & 31 & 2 & 42 & 39 & 40 & & & Total \\
\hline Distâncias & & 200 & 1100 & 1500 & 1300 & 1600 & 52 & 3800 & 1300 & 2100 & 1700 & 850 & & 57907 \\
\hline
\end{tabular}

Fonte: elaborada pelos autores (2020)

Conforme a Tabela 2, a distância percorrida foi de 57907 metros, partindo de uma distância de 210590 metros, a redução neste caso foi de 72,5\% em relação a rota inicial, sendo 183 minutos o tempo para percorrer.

Outras duas tabelas foram feitas: uma utilizando a entrada 2 como saída da entrada 1; e a entrada 1 como saída da entrada 2.

As Tabela 3 e Tabela 4 apresentam os resultados obtidos.

Tabela 3 - Distâncias da Entrada 1, passando pelas 46 escolas e indo para a Saída(entrada) 2

\begin{tabular}{|c|c|c|c|c|c|c|c|c|c|c|c|c|c|c|}
\hline Início & \multicolumn{13}{|c|}{ Google Maps } & \\
\hline Escolas & 47 & 1 & 2 & 3 & 4 & 5 & 6 & 7 & 8 & 9 & 10 & 11 & 12 & \\
\hline Distâncias & & 8500 & 4200 & 1900 & 6800 & 4000 & 4100 & 2300 & 2700 & 5700 & 2400 & 5800 & 1500 & \\
\hline Escolas & 12 & 13 & 14 & 15 & 16 & 17 & \begin{tabular}{|l|}
18 \\
\end{tabular} & 19 & 20 & 21 & 22 & 23 & 24 & \\
\hline Distâncias & & 7600 & 750 & 5400 & 3300 & 7200 & 7300 & 3000 & 5300 & 2500 & 7200 & 3600 & 190 & \\
\hline Escolas & 24 & 25 & 26 & 27 & 28 & 29 & 30 & 31 & 32 & 33 & 34 & 35 & 36 & \\
\hline Distâncias & & 4600 & 4100 & 5800 & 6000 & 7800 & 6400 & 3200 & 3600 & 6400 & 2800 & 7400 & 9600 & \\
\hline Escolas & 36 & 37 & 38 & 39 & 40 & 41 & 42 & 43 & 44 & 45 & 46 & 48 & & Total \\
\hline Distâncias & & 3000 & 5200 & 4200 & 1700 & 5800 & 1900 & 650 & 4400 & 4400 & 5000 & 4600 & & 211790 \\
\hline
\end{tabular}

\begin{tabular}{|c|c|c|c|c|c|c|c|c|c|c|c|c|c|c|}
\hline Final & \multicolumn{13}{|c|}{ Método Evolutionary } & \\
\hline Escolas & 47 & 44 & 6 & 20 & 32 & 17 & 35 & 7 & 21 & 31 & 11 & 12 & 27 & \\
\hline Distâncias & & 1400 & 750 & 650 & 550 & 1700 & 1200 & 1200 & 200 & 2200 & 52 & 1500 & 350 & \\
\hline Escolas & 27 & 3 & 30 & 2 & 39 & 10 & 23 & 24 & 18 & 37 & 15 & 5 & 45 & \\
\hline Distâncias & & 1100 & 25 & 1700 & 2400 & 1200 & 700 & 190 & 800 & 1600 & 160 & 2300 & 550 & \\
\hline Escolas & 45 & 36 & 26 & 38 & 9 & 34 & 42 & 43 & 41 & 25 & 29 & 14 & 1 & \\
\hline Distâncias & & 650 & 1200 & 850 & 1400 & 850 & 1400 & 650 & 1100 & 500 & 2800 & 350 & 95 & \\
\hline Escolas & 1 & 13 & 33 & 22 & 19 & 46 & 4 & 16 & 28 & 40 & 8 & 48 & & Total \\
\hline Distâncias & & 350 & 1000 & 1800 & 130 & 5500 & 500 & 190 & 550 & 3300 & 1600 & 1000 & & 52242 \\
\hline
\end{tabular}

Fonte: elaborada pelos autores (2020) 
WWTERFACEETECNOLOGGCA

Tabela 4 - Distâncias da Entrada 2, passando pelas 46 escolas e indo para a Saída(entrada) 1

\begin{tabular}{|l|c|c|c|c|c|c|c|c|c|c|c|c|c|c|}
\hline Início & \multicolumn{10}{|c|}{ Google Maps } \\
\hline Escolas & 47 & 1 & 2 & 3 & 4 & 5 & 6 & 7 & 8 & 9 & 10 & 11 & 12 & \\
\hline Distâncias & & 7300 & 4200 & 1900 & 6800 & 4000 & 4100 & 2300 & 2700 & 5700 & 2400 & 5800 & 1500 \\
\hline Escolas & 12 & 13 & 14 & 15 & 16 & 17 & 18 & 19 & 20 & 21 & 22 & 23 & 24 \\
\hline Distâncias & & 7600 & 750 & 5400 & 3300 & 7200 & 7300 & 3000 & 5300 & 2500 & 7200 & 3600 & 190 \\
\hline Escolas & 24 & 25 & 26 & 27 & 28 & 29 & 30 & 31 & 32 & 33 & 34 & 35 & 36 \\
\hline Distâncias & & 4600 & 4100 & 5800 & 6000 & 7800 & 6400 & 3200 & 3600 & 6400 & 2800 & 7400 & 9600 & \\
\hline Escolas & 36 & 37 & 38 & 39 & 40 & 41 & 42 & 43 & 44 & 45 & 46 & 48 & & Total \\
\hline Distâncias & & 3000 & 5200 & 4200 & 1700 & 5800 & 1900 & 650 & 4400 & 4400 & 5000 & 6000 & & $\mathbf{2 1 1 9 9 0}$ \\
\hline
\end{tabular}

\begin{tabular}{|c|c|c|c|c|c|c|c|c|c|c|c|c|c|c|}
\hline Final & \multicolumn{13}{|c|}{ Método Evolutionary } & \\
\hline Escolas & 47 & 8 & 40 & 20 & 32 & 6 & 17 & 31 & 11 & 27 & 3 & 30 & 2 & \\
\hline Distâncias & & 1000 & 1500 & 1500 & 550 & 600 & 1800 & 2900 & 52 & 1800 & 1100 & 25 & 1700 & \\
\hline Escolas & 2 & 39 & 10 & 23 & 24 & 18 & 28 & 16 & 46 & 4 & 37 & 15 & 36 & \\
\hline Distâncias & & 2400 & 1200 & 700 & 190 & 800 & 2800 & 550 & 700 & 500 & 2900 & 160 & 2600 & \\
\hline Escolas & 36 & 9 & 34 & 42 & 43 & 41 & 25 & 29 & 14 & 1 & 13 & 33 & 38 & \\
\hline Distâncias & & 1300 & 850 & 1400 & 650 & 1100 & 500 & 2800 & 350 & 95 & 350 & 1000 & 550 & \\
\hline Escolas & 38 & 26 & 19 & 22 & 5 & 45 & 12 & 21 & 7 & 35 & 44 & 48 & & Total \\
\hline Distâncias & & 900 & 1000 & 130 & 1900 & 550 & 4700 & 750 & 200 & 1100 & 2400 & 1300 & & 55902 \\
\hline
\end{tabular}

Fonte: elaborada pelos autores (2020)

Segundo a Tabela 3, a rota otimizada é de 52242 metros, com redução de 75,3\% em relação a rota inicial de 211790 metros. O tempo para percorrer essa distância é de 175 minutos. Na Tabela 4, a otimização resultou em uma distância de 55902 metros, com redução de 73,6\% da rota inicial de 211990 metros, sendo necessários 163 minutos para percorrer.

Comparando as 4 rotas a menor distância foi da Tabela 3 com 52242 metros e tempo de 175 minutos e o menor tempo foi da Tabela 4 com 163 minutos percorrendo 55902 metros. Se for priorizar tempo a escolha deve ser a em relação a rota otimizada da Tabela 4, se for priorizar distância a escolha deve ser em relação a rota otimizada da Tabela 3.

\subsection{Utilizando 2 caminhões para fazer as entregas}

Visto que a rota otimizada de menor tempo foi de 163 minutos, referente a Entrada 2 com a Saída (entrada) 1, que é um tempo considerável uma vez que o caminhão tem que descarregar em 46 escolas, aumentando o tempo total do percurso, foram feitas mais duas tabelas, agora com 2 caminhões. Um caminhão saindo da entrada 1 e passando por 23 escolas, e outro caminhão partindo da entrada 2 e passando por outras 23 escolas.

A Tabela 5 demonstra os resultados atingidos para o caminhão saindo da entrada 1. 


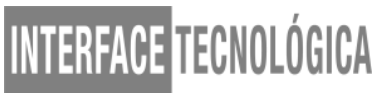

Tabela 5 - Distância, em metros, da entrada 1 com 23 escolas

\begin{tabular}{|c|c|c|c|c|c|c|c|c|c|c|c|c|c|c|}
\hline Início & \multicolumn{13}{|c|}{ Google Maps } & \\
\hline Escolas & 24 & 1 & 2 & 3 & 4 & 5 & 6 & 7 & 8 & 9 & 10 & 11 & 12 & \\
\hline Distâncias & & 1400 & 1800 & 1600 & 2600 & 3000 & 1700 & 2900 & 750 & 200 & 6400 & 550 & 190 & \\
\hline Escolas & 12 & 13 & 14 & 15 & 16 & 17 & 18 & 19 & 20 & 21 & 22 & 23 & & Total \\
\hline Distâncias & & 2800 & 160 & 7800 & 52 & 5900 & 6500 & 6800 & 1800 & 1300 & 2700 & 6100 & 1000 & 66002 \\
\hline
\end{tabular}

\begin{tabular}{|l|c|c|c|c|c|c|c|c|c|c|c|c|c|c|}
\hline Final & \multicolumn{10}{|c|}{ Método Evolutionary } \\
\hline Escolas & 24 & 1 & 3 & 10 & 11 & 18 & 12 & 13 & 14 & 20 & 17 & 21 & 19 & \\
\hline Distâncias & & 1400 & 800 & 4200 & 550 & 700 & 500 & 2800 & 160 & 2700 & 850 & 350 & 800 & \\
\hline Escolas & 19 & 22 & 6 & 5 & 15 & 16 & 7 & 8 & 9 & 2 & 4 & 23 & & Total \\
\hline Distâncias & & 1300 & 2700 & 1900 & 2700 & 52 & 2000 & 750 & 200 & 800 & 1300 & 2800 & 1000 & $\mathbf{3 3 3 1 2}$ \\
\hline
\end{tabular}

Fonte: elaborada pelos autores (2020)

De acordo as Tabela 5, o caminhão saindo pela entrada 1 conseguiu, após a otimização feita pelo Método Evolutionary, diminuir por volta de 50\% a distância a ser percorrida, ou seja, de 66002 metros para 33312 metros, e o tempo que leva para se fazer tal rota é de 86 minutos.

Para a entrada 2, a Tabela 6 expressa os seguintes resultados:

Tabela 6 - Distâncias, em metros, da entrada 2 com 23 escolas

\begin{tabular}{|c|c|c|c|c|c|c|c|c|c|c|c|c|c|c|}
\hline Início & \multicolumn{13}{|c|}{ Google Maps } & \\
\hline Escolas & 24 & 1 & 2 & 3 & 4 & 5 & 6 & 7 & 8 & 9 & 10 & 11 & 12 & \\
\hline Distâncias & & 1100 & 1500 & 650 & 2100 & 1700 & 190 & 4200 & 3900 & 1400 & 3700 & 3300 & 3500 & \\
\hline Escolas & 12 & 13 & 14 & 15 & 16 & 17 & 18 & 19 & 20 & 21 & 22 & 23 & & Total \\
\hline Distâncias & & 5800 & 1700 & 2700 & 350 & 1000 & 1300 & 1700 & 1400 & 4100 & 2900 & 1100 & 6400 & 57690 \\
\hline
\end{tabular}

\begin{tabular}{|c|c|c|c|c|c|c|c|c|c|c|c|c|c|c|}
\hline Final & \multicolumn{13}{|c|}{ Método Evolutionary } & \\
\hline Escolas & 24 & 3 & 2 & 4 & 8 & 12 & 18 & 17 & 19 & 22 & 23 & 20 & 16 & \\
\hline Distâncias & & 1400 & 650 & 1200 & 750 & 1500 & 400 & 750 & 850 & 900 & 1100 & 750 & 2600 & \\
\hline Escolas & 16 & 15 & 11 & 7 & 21 & 14 & 10 & 13 & 5 & 9 & 6 & 1 & & Total \\
\hline Distâncias & & 350 & 100 & 2700 & 650 & 1700 & 650 & 1300 & 2300 & 700 & 800 & 2000 & 850 & 26950 \\
\hline
\end{tabular}

Fonte: elaborada pelos autores (2020)

No que tange a entrada 2 da Tabela 6, o ganho foi de 53,3\%, levando 88 minutos para fazer toda a rota. Nota-se que a distância diminuiu de 57.690 metros, pelo Google Maps, para 26.950 metros após a otimização feita pelo Método Evolutionary, tornando-a a rota de menor distância. Somando as 2 distâncias 33312m mais 26950m é igual a 60262 metros e o tempo será o maior de 88 minutos.

Em termos de economia a melhor opção seria utilizar 1 caminhão conforme a Tabela 3, mas como o tempo de descarregamento deve ser considerado, pode ocorrer de alguma escola ficar sem a entrega dos ingredientes para fazer a merenda no dia seguinte, se isto vier a ocorrer a opção a ser utilizada deve ser de 2 caminhões.

O Quadro 2 demonstra os resultados obtidos em distância e tempo: 


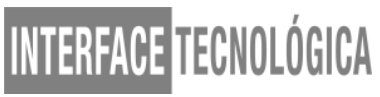

Quadro 2 - Resultados obtidos pelo Método Evolutionary

\begin{tabular}{|l|c|c|}
\hline \multicolumn{1}{|c|}{ 1 Caminhão } & Distância em metros & Tempo em minutos \\
\hline Entrada 1 & 52927 & 177 \\
\hline Entrada 2 & 57907 & 183 \\
\hline Entrada 1 - Saída(entrada) 2 & 52242 & 175 \\
\hline Entrada 2 - Saída(entrada) 1 & 55902 & 163 \\
\hline
\end{tabular}

\begin{tabular}{|c|c|c|}
\hline \multicolumn{1}{|c|}{ 2 Caminhões } & Distância em metros & Tempo em minutos \\
\hline Entrada 1 & 33212 & 86 \\
\hline Entrada 2 & 26950 & 88 \\
\hline
\end{tabular}

Fonte: elaborado pelos autores (2020)

\section{CONSIDERAÇÕES FINAIS}

Após o estudo deste artigo, observa-se que fazer a roteirização por meio do Google Maps não é a maneira mais eficiente e só funciona para até 10 pontos. No entanto, o Google Maps é importante na fase de coleta de dados e para desenhar a rota, além de nos dar a distância que será percorrida e o tempo que levará para fazer o percurso.

Por conta de ter muitas escolas para fazer as entregas, pensou-se também ter mais uma opção para fazer as entregas, que foi a de dividir as escolas para dois caminhões, visto que poderia acontecer atrasos nas entregas, devido ao tempo gasto para percorrer a rota, somando também ao tempo gasto para descarregamento. Em contrapartida, a distância percorrida somando os dois caminhões é maior do que a menor distância percorrida por um caminhão após os resultados da otimização.

Conclui-se com este artigo a eficiência do Método Evolutionary que contempla um número excessivamente maior de pontos que o Google Maps e, após feita as restrições e com o objetivo de minimizar, ele apresenta uma diminuição expressiva nas distâncias a serem percorridas em mais de $70 \%$ com 1 caminhão e por volta de $50 \%$ com dois caminhões, com isso diminuindo consideravelmente o custo e o tempo para as entregas dos ingredientes para fazer a merenda nas 46 escolas da cidade de Barretos.

\section{REFERÊNCIAS}

BALlOU, R. H. Gerenciamento da Cadeia de Suprimentos. 5. ed.: Logística Empresarial. Porto Alegre. Bookman Editora, 2009

BERTAGLIA, P.R. Logística e o gerenciamento da cadeia de abastecimento. 4. ed. São Paulo: Saraiva Educação S.A, 2020. 
BRANDAliSE, L. T. Administração de materiais e logística. Porto Alegre. Simplíssimo Livros Ltda, 2017.

COOPER, D. R.; SCHINDLER, P. S. Métodos de Pesquisa em Administração. 12. ed. Porto Alegre. McGraw Hill Brasil, 2016.

DA FONSECA, R. C. V. Metodologia do Trabalho Científico. Curitiba. IESDE BRASIL S.A., 2016.

DORNIER, P-P. et. al. Logística e Operações Globais: Texto e Casos. 1. ed. 2000/ 6. reimpr. 2007. São Paulo: Atlas, 2007.

FONSECA, J.J.S. Metodologia da Pesquisa Cientifica, 2002. Disponível em: $<$ https://books.google.com.br/books?hl=en\&lr=\&id=oB5x2SChpSEC\&oi=fnd\&pg=PA6\&dq $=$ metodologia + fonseca $+2002 \&$ ots $=$ OROWXq9qh5\&sig $=$ UhQZVC96d3AhLH6xKdUBuUw1 ITY\#v=onepage \&q\&f=false $>$. Acesso em: 17 out. 2020.

FOREMAN, J. W. Data Smart: Usando Data Science para transformar informação em insight. Rio de Janeiro. Alta Books Editora, 2018.

GASPAR-CUNHA, A; TAKAHASHI, R; ANTUNES, C. H. Manual de computação evolutiva e metaheurística. Minas Gerais. UFMG, 2013.

GIL, A.C. Como elaborar projetos de pesquisa. 4. ed. São Paulo, Atlas, 2002

GOULART, V.D.G.; de CAMPOS, A. Logística de Transporte - Gestão Estratégica no Transporte de Cargas. São Paulo: Saraiva Educação S.A, 2018.

HILliER, F. S.; LIEBERMAN, G. J. Introdução à Pesquisa Operacional. 9. ed. Porto Alegre. AMGH Editora LTDA, 2013.

LANES, R. M.; GRECO, M. Aplicação de um método de otimização topológica evolucionária desenvolvido em script python. 2013. Disponível em: $<$ http://www.seer.ufu.br/index.php/cieng/article/view/22636/20069>. Acesso em: 25 Mai 2018.

LOESCH, C.; HEIN, N. Pesquisa Operacional: Fundamentos e modelos. São Paulo. Saraiva Educação S.A., 2017.

LONGARAY, A. A. Introdução à pesquisa operacional. São Paulo. Saraiva Educação S.A., 2017

NOVAES, A. G. Logística e gerenciamento da cadeia de distribuição. 2. ed. Rio de Janeiro. Elsevier, 2007.

PREFEITURA MUNICIPAL DE BARRETOS. Educação Barretos, 2020. Disponível em: $<$ https://www.barretos.sp.gov.br/secretaria-educacao $>$. Acesso em: 12 de mai. de 2020. 
REBOUÇAS, R. S. Problema do Caixeiro Viajante com Coleta de Prêmios e Janelas de Tempo. 2016. Dissertação (Mestrado em Matemática Aplicada) - Instituto de Matemática, Estatística e Computação Científica, Universidade Estadual de Campinas, São Paulo.

SILVA, A.F.; OLIVEIRA, A.C. Algoritmos genéticos: alguns experimentos com os operadores de cruzamento ("Crossover") para o problema do caixeiro viajante assimétrico. XXVI ENEGEP - Fortaleza - CE, 2006. Disponível em: $<$ http://www.abepro.org.br/biblioteca/enegep2006_tr460314_7093.pdf $>$. Acesso em $18 \mathrm{de}$ out de 2020. 